\title{
OPEN Novel use of culturomics to identify the microbiota in hospital sink drains with and without persistent VIM-positive Pseudomonas aeruginosa
}

Jannette Pirzadian ${ }^{1}$, Susan P. Harteveld ${ }^{1,2}$, Shanice N. Ramdutt ${ }^{1,2}$, Willem J. B. van Wamel ${ }^{1}$, Corné H. W. Klaassen ${ }^{1}$, Margreet C. Vos ${ }^{1}$ \& Juliëtte A. Severin ${ }^{1 \bowtie}$

In hospitals, Verona Integron-encoded Metallo-beta-lactamase (VIM)-positive Pseudomonas aeruginosa may colonize sink drains, and from there, be transmitted to patients. These hidden reservoirs are difficult to eradicate since $P$. aeruginosa forms biofilms that resist disinfection. However, little is known on the composition of these biofilms. Therefore, culturomics was used for the first time to investigate the viable microbiota in four hospital sink drain samples with longstanding VIMpositive $P$. aeruginosa drain reservoirs (inhabited by high-risk clone, sequence type ST111), and four drain samples where VIM-positive $P$. aeruginosa was not present. Microbial load and composition varied between samples, yielding between 471-18,904 distinct colonies and 8-20 genera. In two VIMpositive drain samples, $P$. aeruginosa was the most abundantly-isolated microorganism, and found in combination with other Gram-negative bacteria, Citrobacter, Enterobacter, or Stenotrophomonas. P. aeruginosa was in low abundance in the other two VIM-positive samples, and found with Grampositive cocci (Enterococcus and Staphylococcus) or Sphingomonas. In VIM-negative drain samples, high abundances of Gram-negative non-fermenting bacteria, including Acinetobacter, non-aeruginosa Pseudomonas spp., Acidovorax, Chryseobacterium, Flavobacterium, and Sphingobium, as well as Candida, were cultured. Although additional experiments are needed to draw more firm conclusions on which microorganisms enable or inhibit VIM-positive $P$. aeruginosa persistence, our data provide unique insights into the microbial compositions of sink drain inlets.

Hospital plumbing systems provide a significant breeding ground for bacteria. Numerous studies have reported isolating clinically-relevant and opportunistic Gram-negative bacteria from hospital sinks and drain water ${ }^{1}$. Multidrug-resistant (MDR) Pseudomonas aeruginosa has been extensively described in these sites, often in direct patient surroundings ${ }^{1,2}$. P. aeruginosa is well-known for forming biofilms, which hinder disinfection, and allow this bacterium to persist in hospital sink drains or siphons for prolonged periods of time $\mathrm{s}^{3,4}$. Therefore, when carbapenemase-producing, MDR $P$. aeruginosa emerged in hospitals, possible environmental reservoirs and transmission pathways garnered greater focus. Within our tertiary care hospital, Verona Integron-encoded Metallo-beta-lactamase (VIM)-positive P. aeruginosa has been detected at low endemicity in patients since 2003. As part of our prevention efforts, environmental sampling was implemented in 2011, which revealed that sink drains have been acting as persistent environmental sources for these bacteria ${ }^{5,6}$. Through multilocus sequence typing, we determined that within our hospital, the majority of patient- and environment-derived VIM-positive P. aeruginosa isolates were sequence type ST111, a globally-distributed, high-risk clone responsible for extensively drug-resistant nosocomial infections ${ }^{7,8}$. Conversely, environmental sampling also showed that in some sink drains within our hospital, VIM-positive P. aeruginosa has never been cultured before.

During sink use, particles or droplets carrying P. aeruginosa can disperse from sink drains, contaminating the surrounding sink environment and objects used for patient care $\mathrm{e}^{9,10}$; in this way, reservoirs become an

\footnotetext{
${ }^{1}$ Department of Medical Microbiology and Infectious Diseases, Erasmus MC University Medical Center Rotterdam, Rotterdam, The Netherlands. ${ }^{2}$ These authors contributed equally: Susan P. Harteveld and Shanice N. Ramdutt.

${ }$ email: j.severin@erasmusmc.nl
} 
environmental source of transmission for hospital-acquired infections. Recent reports have shown that the design of sinks and the location of drains contribute to the dispersal of these resistant bacteria into the surrounding environment. However, it is currently unknown why VIM-positive P. aeruginosa forms reservoirs and persists in some hospital sink drains, but not in others. We hypothesize that differences in drain microbiota play a role. Antagonism and competition between $P$. aeruginosa and other microorganisms have been previously documented ${ }^{11}$, but these interactions have never yet been described in sink environments.

Sink drain microbiota has been until now unexplored, since in these niches, only MDR bacteria are surveilled. To investigate microbiota, molecular- or culturomics-based methods are used. Culturomics is a technique that uses a diverse repertoire of culture conditions to determine microbial composition ${ }^{12}$. Numerous culture conditions are used to enable growth of minority populations, limit overgrowth by majority populations, and provide insight on the interactions microorganisms experience in a given niche. Culture conditions may also be customized to emulate the environment of origin for difficult-to-culture species, increasing the likelihood of isolation in culture ${ }^{13}$. Compared to molecular methods, culturomics is better able to detect microorganisms in low loads, but detection is limited to viable, cultivable species ${ }^{12}$; however, viable species, especially pathogens, are risks to patients when present in their immediate environment.

Despite its unique advantages, culturomics has never yet been used to study the microbial burden of the innate hospital environment. Therefore, we employed culturomics for the unique application of detecting the viable microbiota in eight separate drain samples from our hospital. We examined four sink drains persistently colonized by VIM-positive $P$. aeruginosa, and four sink drains where VIM-positive $P$. aeruginosa has never been detected before, even after exposure to culture-positive patients. Our aim was to detect majority and minority microbial populations from each sample, and, more specifically, to characterize the microbiota in drains where VIM-positive P. aeruginosa has been either successful or unsuccessful at establishing longstanding reservoirs. By broadening our focus from MDR bacteria to all existing and viable microorganisms, we were able to identify microorganisms that potentially enable or inhibit colonization by VIM-positive P. aeruginosa. This knowledge may contribute to the development of environmental probiotic treatments to decolonize future sink drains from these notoriously persistent bacteria, or prevent future reservoir formation.

\section{Methods}

Setting. The Erasmus MC University Medical Center is a tertiary care hospital in Rotterdam, the Netherlands. Adult intensive care units consisted of single-patient rooms, some with anterooms. The general surgery and gastrointestinal surgery wards consisted of two- and four-bed patient rooms. Environmental sampling for VIM-positive $P$. aeruginosa occurred sporadically before 2011, and systematically after 2011 following increasing numbers of VIM-positive $P$. aeruginosa patients. Indications for environmental sampling were (1) unexpected colonization of a patient in either the room or ward, and (2) follow-up after an environmental intervention, such as when drain plugs were installed in the sinks from six wards (J. A. Severin, unpublished data). Until patient wards closed in May 2018 for demolition, VIM-positive P. aeruginosa was frequently cultured from the environment, including from the positive sink drains chosen for this study.

As this study did not involve humans, animals, or interventions, in accordance with Dutch law, neither ethical approval nor waiving of ethical approval was required. Furthermore, all methods described were carried out under relevant guidelines and regulations.

Sink selection and sample collection. Previous environmental sampling data were used to select sinks. Only sinks from wards most affected by VIM-positive $P$. aeruginosa colonization were considered for inclusion. Four positive sinks (represented by ${ }^{+}$) were chosen that were considered hotspots for VIM-positive P. aeruginosa over a period of at least four years (Supplementary Fig. S1). Four negative sinks (represented by ${ }^{-}$) were chosen where VIM-positive $P$. aeruginosa had not been cultured over a period of four years, even after exposure to use by or waste from admitted, culture-positive patients (Supplementary Fig. S1). On average, 1.5 environmental cultures were taken per sink per year. Immediately prior to each culturomics experiment, the sink environment was sampled again: the wash basin, counter, faucet aerator, and drain inlet were swabbed (BBL CultureSwab Plus, BD Diagnostics, Sparks, MD, United States). Swabs were incubated overnight at $35^{\circ} \mathrm{C}$ in $5 \mathrm{ml}$ of a CAZVAN enrichment broth consisting of tryptic soy broth with $2 \mathrm{mg} / \mathrm{l}$ ceftazidime (CAZ) and $50 \mathrm{mg} / \mathrm{l}$ vancomycin (VAN). From this broth, DNA was extracted using MagNa Pure 96 (Roche Diagnostics, Almere, the Netherlands) according to the manufacturer's instructions. DNA was then screened for $b l a_{\mathrm{VIM}}$ in-house using real-time PCR: $5 \mu \mathrm{l} \mathrm{DNA}$ were added to $5 \mu \mathrm{l}$ primer/probe mix [forward primer 5'-GCAAATTGGACTTCCYGTAA-3', reverse primers 5'-GACGGTGATGCGTACGTTG-3' and 5'-CCCTAAGGGCATCAACTCC-3', probe 5'-Cy5TTTCATGACGACCGCGTCGG-3'-BHQ-2 (Eurogentec, Maastricht, the Netherlands)] and $10 \mu$ LightCycler 480 Probes Master (Roche Diagnostics, Almere, the Netherlands). PCR was performed in a LightCycler 480 Real-Time PCR Instrument (Roche Diagnostics, Almere, the Netherlands) using the following program: predenaturation $\left(95^{\circ} \mathrm{C} / 5 \mathrm{~min}\right)$, followed by 50 cycles of denaturation $\left(95^{\circ} \mathrm{C} / 5 \mathrm{~s}\right)$ and annealing $\left(60^{\circ} \mathrm{C} / 30 \mathrm{~s}\right)$. VIMpositive $P$. aeruginosa strain S04 90 was used as a positive control ${ }^{14}$.

Drain plugs (article no. UD.526.51, Raminex, Utrecht, the Netherlands) from the eight selected sinks were aseptically removed and stored in separate 1.11 sterile sonication containers. Drain plugs were entirely comprised of stainless steel, and were all of identical size and design (Fig. 1).

Culturomics. Drain samples were obtained by sonicating drain plugs to dislodge biofilms. We hypothesized that drain plugs were contaminated by the same microorganisms colonizing the upper ends of siphons and drain inlets, and that these microorganisms were most at risk of dispersal from sink use. Before sonicating, the underside of each drain plug was scraped with a sterile scalpel to sample the topmost layer of biofilm. The scalpel was 


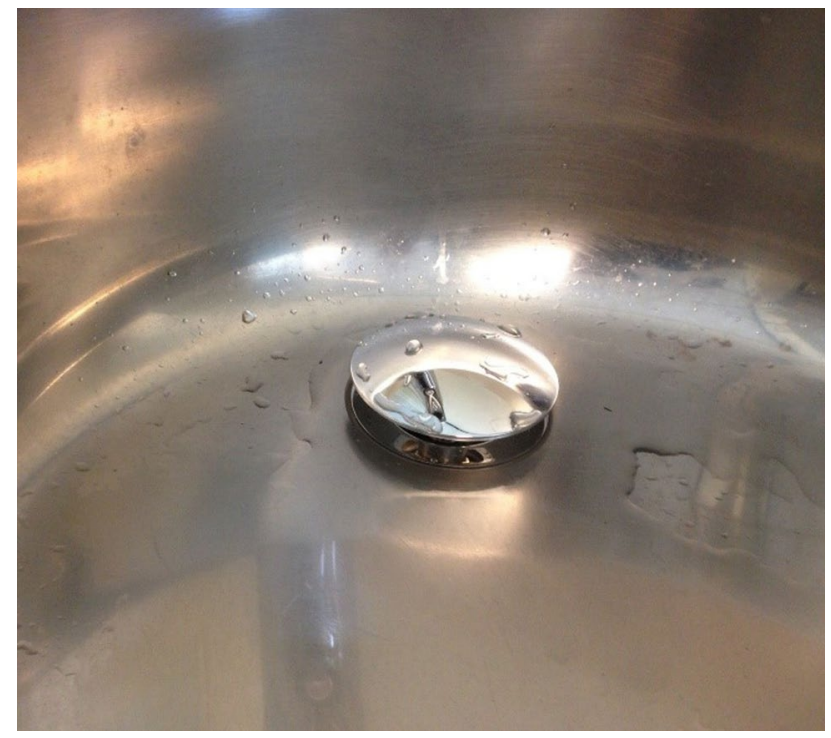

Figure 1. Drain plugs used in this study. Only sinks containing identical stainless steel drain plugs were considered for inclusion. The surface of these plugs contained no holes, and when screwed into the drain, completely covered the drain inlet.

consecutively pressed onto four Tryptic Soy Agar II with 5\% Sheep Blood (TSA) plates (BD Diagnostics, Breda, the Netherlands). Each of the four plates was incubated under a different condition: one at $35^{\circ} \mathrm{C}$ in aerobic conditions, one at $35^{\circ} \mathrm{C}$ in aerobic conditions with $5 \% \mathrm{CO}_{2}$, one at $35^{\circ} \mathrm{C}$ in microaerophilic conditions, and one at $35^{\circ} \mathrm{C}$ in anaerobic conditions (Table 1 , Supplementary Table S2).

Subsequently, drain plugs were subjected to sonication using the protocol for orthopedic prostheses to diagnose prosthesis-associated infections: the drain plug was covered in $115 \mathrm{ml} 0.9 \% \mathrm{NaCl}$, scraped, shaken rigorously for $30 \mathrm{~s}$ inside the sonication container, and sonicated for $1 \mathrm{~min}$ at $40 \mathrm{kHz}, 800 \mathrm{~W}$ at $100 \%$ power in an ultrasonic water bath (Bandelin Electronic, Berlin, Germany) ${ }^{15}$. Following sonication, the container was shaken rigorously again for $30 \mathrm{~s}$. In a laminar flow cabinet, $100 \mu \mathrm{l}$ of sonication fluid were inoculated into $5 \mathrm{ml}$ of each broth, and onto every agar plate using disposable spreaders; $3 \mathrm{ml}$ of sonication fluid were inoculated into each blood culture bottle (BD BACTEC, BD Diagnostics, Breda, the Netherlands) with sterile syringes and needles. Culturomics experiments were independently performed for each drain sample.

Culture media were chosen from a repertoire of routine and specialized diagnostic culture media, because the likelihood of cultivating human commensal flora and pathogens was high (Table 1, Supplementary Table S2). Several conditions used were inspired by the seminal culturomics article by Lagier et al.: active filtration of sonication fluid through $0.45 \mu \mathrm{m}$-pore-sized filters was performed prior to inoculation to isolate smaller cell sizes; thermic shock pretreatment was performed by submerging sonication fluid in an $80^{\circ} \mathrm{C}$ water bath for $20 \mathrm{~min}$ prior to inoculation to isolate existing spores; different atmospheric conditions were used to differentiate by oxygen requirement; blood culture enrichment followed by subculture on TSA was used to stimulate the growth of clinical isolates; antibiotic-supplemented media were used to isolate MDR species; eosin methylene blue agar was used to isolate enteric microorganisms; Reasoner's 2A agar was used to isolate water-associated microorganisms; and cetrimide agar was used to select for Pseudomonas spp. (Table 1, Supplementary Table S2) ${ }^{12}$. In addition to incubation at $35^{\circ} \mathrm{C}$, media were also incubated at room temperature to mimic sink environment temperatures (Table 1, Supplementary Table S2). Finally, a custom agar medium was created by our laboratory: Mueller Hinton II Agar (BD Diagnostics, Breda, the Netherlands) was made according to the manufacturer's instructions, but purified water was replaced with tap water from the tested wards (Table 1, Supplementary Table S2).

Then, the drain plug was aseptically transferred to another sterile sonication container with $115 \mathrm{ml} 0.9 \% \mathrm{NaCl}$, scraped, shaken for $30 \mathrm{~s}$, and sonicated for an additional $4 \mathrm{~min}$ ( 5 cumulative min sonication time) to retrieve viable microorganisms potentially present in deeper layers of biofilm that were not dislodged during the initial sonication step ${ }^{16,17}$. This container was shaken again for $30 \mathrm{~s}$, and $100 \mu \mathrm{l}$ were respectively inoculated onto one TSA and one Brucella agar plate (BD Diagnostics, Breda, the Netherlands) that were incubated aerobically at $35^{\circ} \mathrm{C}$, and onto one TSA and one Brucella agar plate that were incubated anaerobically at $35^{\circ} \mathrm{C}$ (Table 1 , Supplementary Table S2). In total, 179 culture conditions were tested per drain sample (Table 1, Supplementary Table S2). All commercially-prepared culture media were provided by BD Diagnostics, Breda, the Netherlands or Thermo Fisher Scientific, Bleiswijk, the Netherlands. For culture media created in-house, materials were supplied by BD Diagnostics, Breda, the Netherlands or Sigma-Aldrich Chemie, Zwijndrecht, the Netherlands.

Growth characterization and quantification. Cultures were checked regularly for growth, and incubated for a total of 6 weeks or until nearly confluent. Morphologically-unique colonies that were visible to the unaided eye were subcultured onto TSA or onto the medium on which they initially grew. Rapid identification of isolates was performed using MALDI-TOF MS (Bruker Daltonik, Bremen, Germany) according to the manu- 


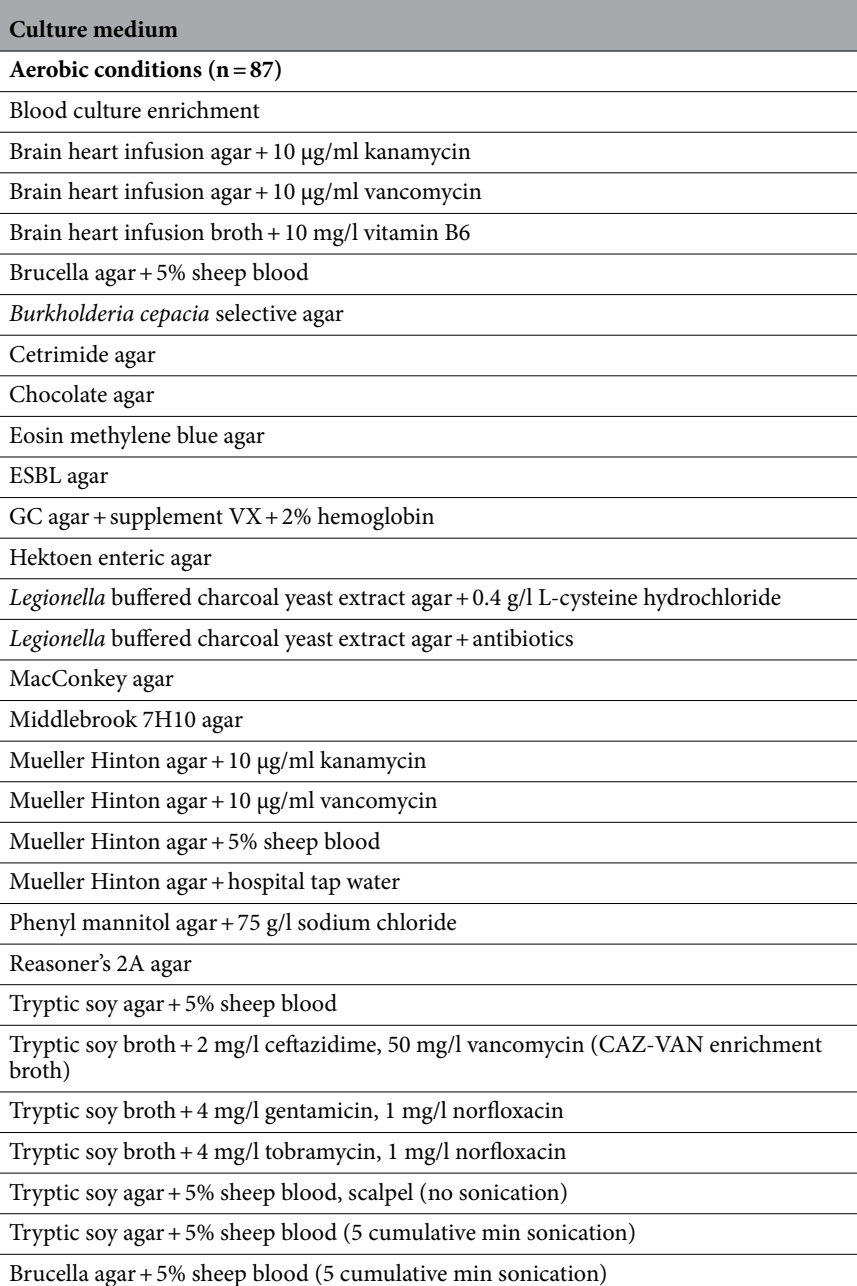

\section{Microaerophilic conditions $(n=47)$}

Brain heart infusion agar $+10 \mu \mathrm{g} / \mathrm{ml}$ kanamycin

Brain heart infusion agar $+10 \mu \mathrm{g} / \mathrm{ml}$ vancomycin

Brucella agar $+5 \%$ sheep blood

Burkholderia cepacia selective agar

Cetrimide agar

Chocolate agar

Eosin methylene blue agar

ESBL agar

GC agar + supplement VX $+2 \%$ hemoglobin

Hektoen enteric agar

Legionella buffered charcoal yeast extract agar $+0.4 \mathrm{~g} / \mathrm{l}$ L-cysteine hydrochloride

Legionella buffered charcoal yeast extract agar + antibiotics

MacConkey agar

Middlebrook 7H10 agar

Mueller Hinton agar $+10 \mu \mathrm{g} / \mathrm{ml}$ kanamycin

Mueller Hinton agar $+10 \mu \mathrm{g} / \mathrm{ml}$ vancomycin

Mueller Hinton agar $+5 \%$ sheep blood

Mueller Hinton agar + hospital tap water

Phenyl mannitol agar $+75 \mathrm{~g} / \mathrm{l}$ sodium chloride

Reasoner's 2A agar

Tryptic soy agar $+5 \%$ sheep blood

Tryptic soy agar $+5 \%$ sheep blood, scalpel (no sonication)

Anaerobic conditions $(n=45)$

Bacteroides bile esculin agar

Continued

\begin{tabular}{|c|c|c|c|c|c|c|}
\hline$X$ & $X$ & $x$ & $X$ & $X$ & $x$ & \\
\hline$x$ & & & $x$ & & & $x$ \\
\hline$x$ & & & $x$ & & & $x$ \\
\hline$x$ & & & $x$ & & & $X$ \\
\hline$x$ & & & $x$ & & & $X$ \\
\hline$x$ & & & $x$ & & & $X$ \\
\hline$x$ & & & $x$ & & & $X$ \\
\hline$x$ & & & $x$ & & & $X$ \\
\hline$x$ & & & $x$ & & & $X$ \\
\hline$x$ & & & $x$ & & & $x$ \\
\hline$x$ & & & $x$ & & & $x$ \\
\hline$x$ & & & $x$ & & & $X$ \\
\hline$x$ & & & $x$ & & & \\
\hline$x$ & & & $x$ & & & \\
\hline$x$ & & & $x$ & & & $x$ \\
\hline$x$ & & & $x$ & & & $x$ \\
\hline$x$ & & & $x$ & & & $\times$ \\
\hline$x$ & & & $x$ & & & $X$ \\
\hline$x$ & & & $x$ & & & $\times$ \\
\hline$x$ & & & $x$ & & & $X$ \\
\hline$x$ & & & $x$ & & & $X$ \\
\hline$x$ & & & $x$ & & & $X$ \\
\hline$x$ & $x$ & $x$ & $x$ & $x$ & $x$ & $X$ \\
\hline$X$ & & & $x$ & & & $\times$ \\
\hline$x$ & & & $x$ & & & $X$ \\
\hline \multirow[t]{4}{*}{$x$} & & & $x$ & & & $x$ \\
\hline & & & $x$ & & & $x$ \\
\hline & & & $x$ & & & \\
\hline & & & $x$ & & & \\
\hline
\end{tabular}

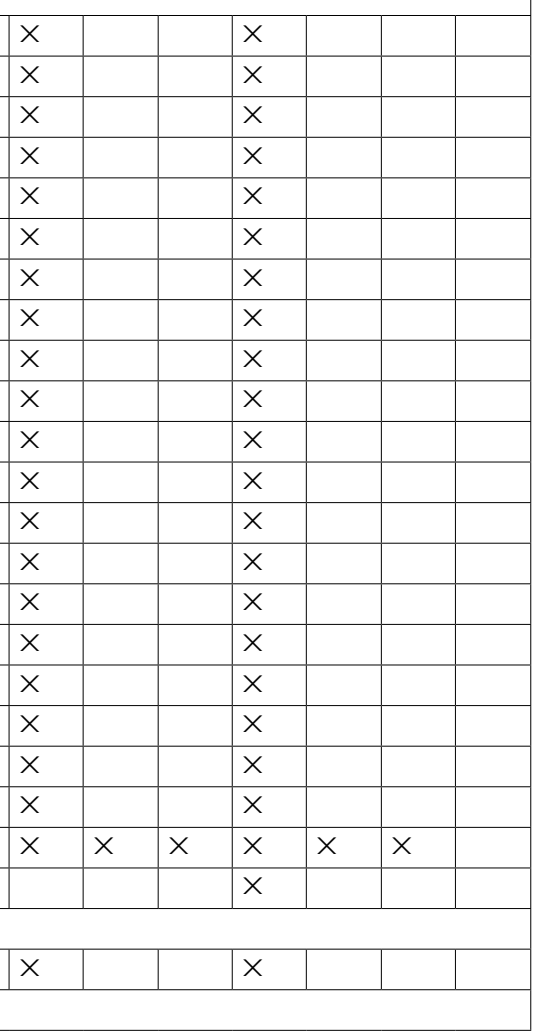




\begin{tabular}{|c|c|c|c|c|c|c|c|}
\hline \multirow[b]{2}{*}{ Culture medium } & \multicolumn{3}{|c|}{ Room temperature } & \multicolumn{4}{|c|}{$35^{\circ} \mathrm{C}$} \\
\hline & & 0.45 & TS & & 0.45 & TS & $\mathrm{CO}_{2}$ \\
\hline Blood culture enrichment & $x$ & $x$ & $x$ & $\times$ & $x$ & $x$ & \\
\hline Brain heart infusion agar $+10 \mu \mathrm{g} / \mathrm{ml}$ kanamycin & $x$ & & & $\times$ & & & \\
\hline Brain heart infusion agar $+10 \mu \mathrm{g} / \mathrm{ml}$ vancomycin & $x$ & & & $\times$ & & & \\
\hline Brucella agar $+5 \%$ sheep blood & $x$ & $x$ & $x$ & $\times$ & $x$ & $x$ & \\
\hline CDC anaerobe $5 \%$ sheep blood agar + phenylethyl alcohol & $x$ & & & $\times$ & & & \\
\hline Cetrimide agar & $x$ & & & $\times$ & & & \\
\hline Chocolate agar & $x$ & & & $\times$ & & & \\
\hline ESBL agar & $x$ & & & $\times$ & & & \\
\hline MacConkey agar & $x$ & & & $\times$ & & & \\
\hline Mueller Hinton agar $+10 \mu \mathrm{g} / \mathrm{ml}$ kanamycin & $x$ & & & $\times$ & & & \\
\hline Mueller Hinton agar $+10 \mu \mathrm{g} / \mathrm{ml}$ vancomycin & $x$ & & & $\times$ & & & \\
\hline Mueller Hinton agar $+5 \%$ sheep blood & $x$ & & & $\times$ & & & \\
\hline Mueller Hinton agar + hospital tap water & $x$ & & & $\times$ & & & \\
\hline Tryptic soy agar $+5 \%$ sheep blood & $x$ & $x$ & $x$ & $\times$ & $x$ & $x$ & \\
\hline Tryptic soy agar $+5 \%$ sheep blood, scalpel (no sonication) & & & & $\times$ & & & \\
\hline Tryptic soy agar $+5 \%$ sheep blood ( 5 cumulative min sonication) & & & & $\times$ & & & \\
\hline Brucella agar $+5 \%$ sheep blood ( 5 cumulative min sonication) & & & & $\times$ & & & \\
\hline
\end{tabular}

Table 1. Overview of culturomics culture media and conditions. In the table, a cross mark means that the indicated condition was tested for that culture medium. $0.45=$ sonication fluid was subjected to $0.45 \mu \mathrm{m}$-sized pore prefiltration prior to inoculation. $\mathrm{TS}=$ sonication fluid was subjected to thermic shock pretreatment prior to inoculation. $\mathrm{CO}_{2}=$ media were incubated at $35^{\circ} \mathrm{C}$ in aerobic conditions with $5 \% \mathrm{CO}_{2}$. CDC Centers for Disease Control and Prevention; ESBL extended spectrum beta-lactamase; GC Gonococcal; CAZ ceftazidime; VAN vancomycin.

facturer's instructions. Genus identification was accepted for scores above 1.80, and species identification was accepted for scores above 2.0. In case MALDI-TOF MS could not identify a microorganism to the genus level, identification was performed by partial $16 \mathrm{~S}$ rRNA gene sequencing.

To calculate relative abundances per drain sample, total colony counts were enumerated for each genus. Growth in broths and blood culture bottles was uncountable and not included; growth in these conditions was only used to document the presence/absence of microorganisms not found in solid media.

From each drain sample, a selection of $P$. aeruginosa isolates and other Gram-negative bacteria were screened for $b l a_{\mathrm{VIM}}$ using PCR as described above. In case few Gram-negative bacteria were found, all Gram-negative isolates were then screened for $b l a_{\mathrm{VIM}}$.

16S rRNA gene sequencing. To identify microorganisms not identifiable by MALDI-TOF MS, DNA was isolated as described above, and sequenced targeting the first $\sim 550$ bp of the $16 \mathrm{~S}$ rRNA gene. For isolates that could not be subcultured from original cultures, DNA was extracted by picking a colony from an original culture using a toothpick, and suspending the inoculum in $25 \mu \mathrm{l}$ water and $5 \mu$ proteinase $\mathrm{K}$ before heating $\left(56^{\circ} \mathrm{C} / 10 \mathrm{~min}\right.$ followed by $99^{\circ} \mathrm{C} / 5 \mathrm{~s}$ ). Briefly, $10 \mu \mathrm{LNA}$ were added to $2.5 \mu \mathrm{l} 10 \times$ primer mix [ $5 \mu \mathrm{M}$ each of forward primer 5'-AGAGTTTGATCMTGGYTCAG-3' and reverse primer 5'-CTTTACGCCCARTRAWTCCG-3' (Eurogentec, Maastricht, the Netherlands)] and $12.5 \mu \mathrm{l}$ FastStart PCR Master (Roche Diagnostics, Almere, the Netherlands). PCR was performed in a thermocycler using the following program: pre-denaturation $\left(95^{\circ} \mathrm{C} / 5 \mathrm{~min}\right)$, followed by 20 cycles of denaturation $\left(94^{\circ} \mathrm{C} / 30 \mathrm{~s}\right)$, annealing $\left(55^{\circ} \mathrm{C} / 30 \mathrm{~s}\right)$, and extension $\left(72^{\circ} \mathrm{C} / 1 \mathrm{~min}\right)$. Prior to sequencing, $20 \mu \mathrm{l}$ of PCR product were treated with ExoSAP-IT Express PCR Product Cleanup Reagent (Thermo Fisher Scientific, Bleiswijk, the Netherlands) using the recommended conditions. Sequencing was performed by BaseClear, Leiden, the Netherlands. A standard BLAST analysis was performed using the obtained DNA sequences to identify the microorganisms involved.

Legionella and Acanthamoeba PCRs. To detect the presence of Legionella spp. or Acanthamoeba spp. in drain samples, $10 \mathrm{ml}$ from both 1- and 5-min sonication fluids were centrifuged at $4000 \mathrm{rpm} / 15 \mathrm{~min}$ to pellet cells, and the supernatants were decanted. Cells were resuspended in residual sonication fluid to make concentrated suspensions, and then DNA extraction was performed as described above. For Acanthamoeba PCR, a bead beating step (30 s at a frequency of $30 \mathrm{r} / \mathrm{s}$ ) preceded DNA extraction. For the detection of Legionella spp., an in-house real-time PCR was performed based on the assay described by Templeton et al. with minor modifications ${ }^{18}$; L. pneumophila CCUG 33,152 was used as a positive control. For the detection of Acanthamoeba spp., an in-house real-time PCR was performed based on the assay described by Qvarnstrom et al. with minor modifications ${ }^{19}$; A. castellanii CCAP $1501 / 1 \mathrm{~B}$ was used as a positive control. 


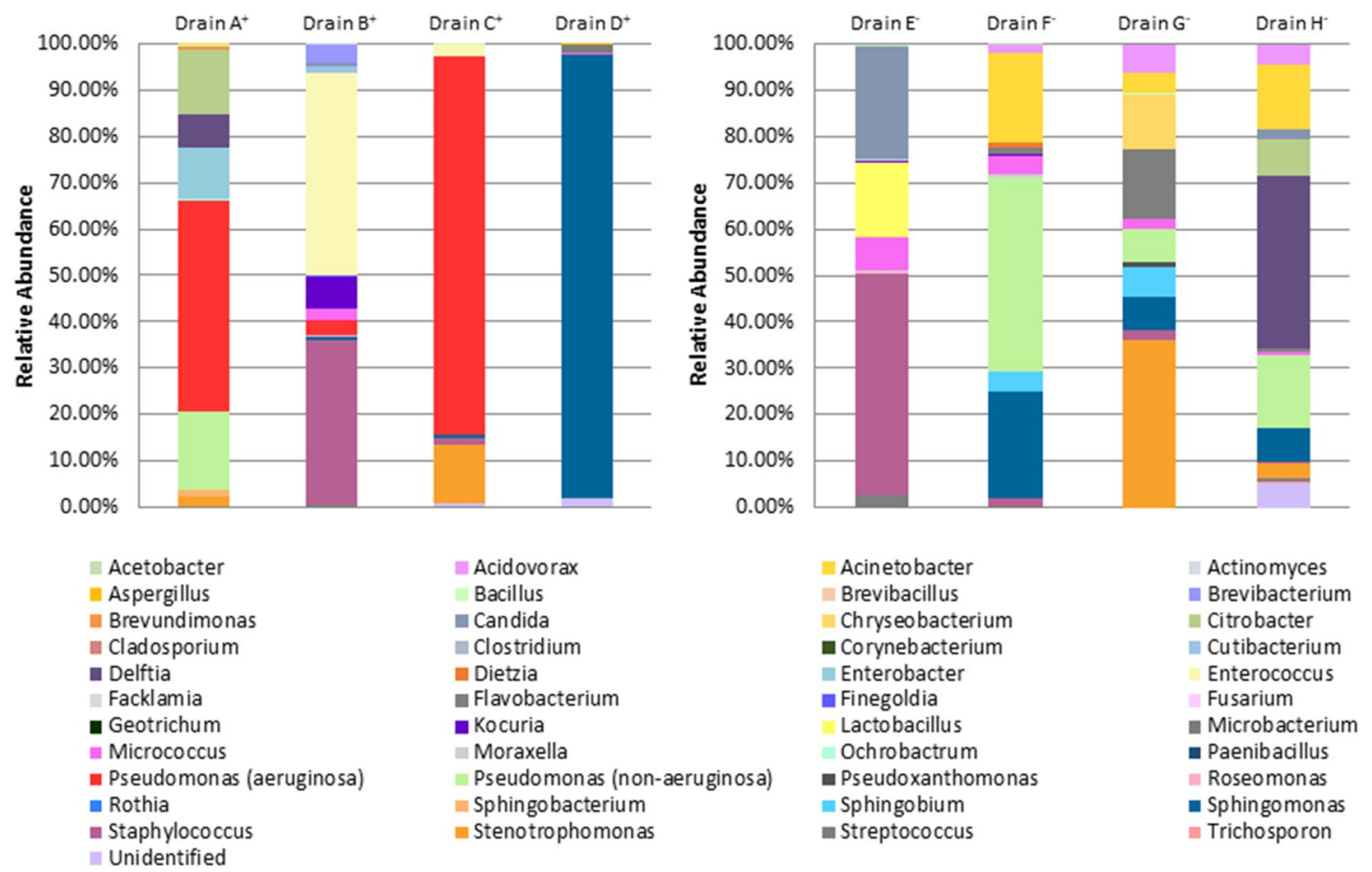

Figure 2. Relative abundance of all genera. Drains $\mathrm{A}^{+}, \mathrm{B}^{+}, \mathrm{C}^{+}$, and $\mathrm{D}^{+}$were sink drain samples containing VIMpositive P. aeruginosa. Drains $\mathrm{E}^{-}, \mathrm{F}^{-}, \mathrm{G}^{-}$, and $\mathrm{H}^{-}$were sink drain samples that did not contain VIM-positive $P$. aeruginosa. Relative abundances are shown as percentages.

\section{Results}

Sink selection. Among positive sinks that were selected based on persistent colonization by VIM-positive $P$. aeruginosa, three were from patient rooms in adult intensive care units (Drains $\mathrm{A}^{+}, \mathrm{C}^{+}$, and $\mathrm{D}^{+}$), while the fourth sink was from a patient room in the gastrointestinal surgery ward (Drain $\mathrm{B}^{+}$) (Supplementary Fig. S1). PCR on swab cultures immediately prior to each culturomics experiment confirmed the presence of VIM-positive $P$. aeruginosa in these sinks' drains.

Among negative sinks that were selected based on never having cultured VIM-positive P. aeruginosa in the preceding four-year period, one was from a patient room anteroom in adult intensive care (Drain $\mathrm{E}^{-}$), one from a patient room in the general surgery ward $\left(\right.$ Drain $\mathrm{F}^{-}$), one from a communal patient bathroom in the gastrointestinal surgery ward (Drain $\mathrm{G}^{-}$), and one from a dirty utility room for healthcare workers in the general surgery ward (Drain $\mathrm{H}^{-}$) (Supplementary Fig. S1). PCR on swab cultures immediately prior to each culturomics experiment confirmed the absence of VIM-positive $P$. aeruginosa in these sinks' drains. The anteroom with a negative sink (Drain $\mathrm{E}^{-}$) adjoined a patient room with a positive sink (Drain $\mathrm{C}^{+}$); additionally, the wastewater pipelines of these sinks were connected.

Culturomics. Culturomics was performed on drain samples obtained from the eight selected sinks. Results are presented in Fig. 2 and Table 2, while a list of identifiable species is presented in Supplementary Table S3. In the four positive sink drain samples, VIM-positive $P$. aeruginosa was successfully cultured using culturomics, but the relative bacterial load differed; in two samples (Drains $\mathrm{A}^{+}$and $\mathrm{C}^{+}$), VIM-positive P. aeruginosa was the most abundantly-isolated microorganism, accounting for $46 \%$ and $81 \%$ of all colonies, respectively. From Drain $\mathrm{A}^{+}$, we also observed moderate relative abundances of Citrobacter (14\%), Delftia (7\%), Enterobacter (11\%), and Pseudomonas putida group isolates (17\%), specifically P. putida and P. monteilii (Supplementary Table S3). From Drain $\mathrm{C}^{+}$, only Stenotrophomonas was in moderate relative abundance (13\%), while other genera appeared suppressed due to the overrepresentation of $P$. aeruginosa. Fewer than $1 \%$ of colonies from Drain $\mathrm{C}^{+}$were unidentifiable (Table 2). In the two other positive sink drain samples (Drains $\mathrm{B}^{+}$and $\mathrm{D}^{+}$), VIM-positive P. aeruginosa was cultured, but in lower relative abundance compared to other genera. Drain $\mathrm{B}^{+}$primarily grew Enterococcus $(44 \%)$ and coagulase-negative staphylococci (36\%), while P. aeruginosa was only detected among 3\% of colonies. Drain $\mathrm{D}^{+}$was dominated by growth of Sphingomonas (96\%), while $P$. aeruginosa was found among $<0.1 \%$ of colonies. Two percent of colonies from Drain $\mathrm{D}^{+}$were unidentifiable (Table 2).

In two out of four negative sink drain samples, VIM-negative $P$. aeruginosa isolates were found (Drains $\mathrm{F}^{-}$and $\mathrm{H}^{-}$), but no other $P$. aeruginosa was cultured (Table 2). Drain $\mathrm{E}^{-}$grew moderate relative abundances of Candida (24\%), Lactobacillus (16\%), and Micrococcus (7\%), and a high relative abundance of coagulase-negative staphylococci ( $48 \%$ of colonies). This drain was physically near and closely connected to Drain $\mathrm{C}^{+}$, but the drain microbiota between these samples showed major differences: only Bacillus, Micrococcus, and Staphylococcus were 


\begin{tabular}{|c|c|c|c|c|c|c|c|c|}
\hline \multirow[b]{3}{*}{ Genus } & \multicolumn{8}{|c|}{$\%$} \\
\hline & \multicolumn{4}{|c|}{ VIM-positive sink drain samples } & \multicolumn{4}{|c|}{ VIM-negative sink drain samples } \\
\hline & $\begin{array}{c}\text { Drain } \\
\mathrm{A}^{+}\end{array}$ & $\begin{array}{c}\text { Drain } \\
\mathrm{B}^{+}\end{array}$ & $\begin{array}{c}\text { Drain } \\
\mathrm{C}^{+}\end{array}$ & $\begin{array}{c}\text { Drain } \\
\mathrm{D}^{+}\end{array}$ & $\begin{array}{c}\text { Drain } \\
E\end{array}$ & $\begin{array}{c}\text { Drain } \\
\mathrm{F}^{-}\end{array}$ & $\begin{array}{c}\text { Drain } \\
G^{-}\end{array}$ & $\begin{array}{c}\text { Drain } \\
\mathrm{H}^{-}\end{array}$ \\
\hline \multicolumn{9}{|c|}{ Gram-negative non-fermenting bacteria } \\
\hline $\begin{array}{c}\text { Pseudomonas } \\
\text { aeruginosa }\end{array}$ & 45.51 & 3.41 & 81.47 & 0.04 & 0.00 & 0.01 & 0.00 & $\S$ \\
\hline $\begin{array}{c}\text { Non-aeruginosa } \\
\text { Pseudomonas spp. }\end{array}$ & 16.63 & 0.00 & 0.00 & 0.00 & 0.00 & 42.08 & 7.23 & 15.55 \\
\hline Achromobacter & $\S$ & 0.00 & 0.00 & 0.00 & 0.00 & 0.00 & 0.00 & 0.00 \\
\hline Acidovorax & 0.00 & 0.00 & 0.00 & 0.00 & 0.00 & 1.82 & 6.23 & 4.30 \\
\hline Acinetobacter & 0.27 & 0.00 & 0.00 & 0.00 & 0.00 & 19.43 & 4.22 & 14.22 \\
\hline Brevundimonas & 0.48 & 0.00 & 0.00 & 0.00 & 0.00 & 0.00 & 0.00 & 0.00 \\
\hline Chryseobacterium & 0.00 & 0.00 & 0.00 & 0.00 & 0.00 & 0.00 & 11.49 & 0.02 \\
\hline Cupriavidus & 0.00 & 0.00 & 0.00 & 0.00 & 0.00 & 0.00 & $\S$ & 0.00 \\
\hline Delftia & 7.06 & 0.00 & 0.00 & 0.00 & 0.00 & 0.00 & 0.00 & 37.27 \\
\hline Flavobacterium & 0.00 & 0.00 & 0.00 & 0.00 & 0.00 & 1.12 & 15.08 & 0.66 \\
\hline Moraxella & 0.00 & 0.13 & 0.00 & 0.00 & 0.21 & 0.56 & 0.00 & 0.00 \\
\hline Ochrobactrum & 0.30 & 0.00 & 0.00 & 0.00 & 0.00 & 0.00 & 0.00 & 0.00 \\
\hline Roseomonas & 0.00 & 0.06 & 0.00 & 0.00 & 0.21 & 0.00 & 0.00 & 0.00 \\
\hline Sphingobacterium & 1.50 & 0.00 & 0.00 & 0.00 & 0.00 & 0.00 & 0.00 & 0.00 \\
\hline Sphingobium & 0.00 & 0.00 & 0.00 & 0.00 & 0.00 & 4.13 & 6.59 & 0.02 \\
\hline Sphingomonas & 0.00 & 0.70 & 0.71 & 95.95 & 0.00 & 22.98 & 7.10 & 7.44 \\
\hline Stenotrophomonas & 2.21 & 0.00 & 12.80 & 0.00 & 0.00 & 0.05 & 35.97 & 3.53 \\
\hline \multicolumn{9}{|c|}{ Other Gram-negative bacteria } \\
\hline Acetobacter & 0.00 & 0.00 & 0.00 & 0.00 & 0.00 & 0.01 & 0.00 & 0.00 \\
\hline Citrobacter & 14.20 & 0.00 & 0.00 & 0.00 & 0.00 & 0.00 & 0.00 & 8.07 \\
\hline Enterobacter & 11.15 & 0.00 & 0.00 & 0.00 & 0.00 & 0.00 & 0.00 & 0.06 \\
\hline Pseudoxanthomonas & 0.00 & 0.00 & 0.00 & 0.00 & 0.00 & 0.00 & 0.79 & 0.00 \\
\hline \multicolumn{9}{|c|}{ Gram-positive bacteria } \\
\hline Actinomyces & 0.00 & 0.06 & 0.00 & 0.00 & 0.00 & 0.00 & 0.00 & 0.00 \\
\hline Bacillus & 0.06 & 0.25 & 0.01 & 0.00 & 0.42 & 0.01 & 0.62 & 0.00 \\
\hline Brevibacillus & 0.24 & 0.00 & 0.00 & 0.00 & 0.00 & 0.00 & 0.00 & 0.00 \\
\hline Brevibacterium & 0.00 & 3.86 & 0.00 & 0.02 & 0.00 & 0.02 & 0.00 & 0.00 \\
\hline Clostridium & 0.00 & 0.00 & 0.00 & 0.00 & 0.00 & 0.00 & 0.00 & 0.01 \\
\hline Corynebacterium & 0.00 & 0.13 & 0.00 & 0.00 & 0.00 & 0.00 & 0.00 & 0.00 \\
\hline Cutibacterium & 0.00 & 1.39 & 0.00 & 0.00 & 0.21 & 0.00 & 0.10 & 0.01 \\
\hline Dietzia & 0.00 & 0.00 & 0.00 & 0.00 & 0.00 & 1.10 & 0.00 & 0.00 \\
\hline Enterococcus & 0.21 & 43.93 & 2.64 & 0.00 & 0.00 & 0.00 & 0.11 & 0.00 \\
\hline Facklamia & 0.00 & 0.00 & 0.00 & 0.00 & 0.21 & 0.00 & 0.00 & 0.00 \\
\hline Finegoldia & 0.00 & 0.25 & 0.00 & 0.00 & 0.00 & 0.00 & 0.00 & 0.00 \\
\hline Kocuria & 0.00 & 6.89 & 0.00 & 0.02 & 0.42 & 0.55 & 0.00 & 0.00 \\
\hline Lactobacillus & 0.00 & 0.00 & 0.00 & 0.00 & 15.92 & 0.00 & 0.00 & 0.00 \\
\hline Microbacterium & 0.00 & 0.00 & 0.00 & 1.78 & 0.00 & 0.00 & 0.00 & 0.00 \\
\hline Micrococcus & 0.00 & 2.28 & 0.16 & 0.29 & 7.43 & 4.00 & 2.20 & 0.70 \\
\hline Paenibacillus & 0.03 & 0.00 & 0.00 & 0.00 & 0.00 & 0.01 & 0.01 & 0.01 \\
\hline Rothia & 0.00 & 0.00 & 0.00 & 0.00 & 0.00 & 0.01 & 0.00 & 0.00 \\
\hline Staphylococcus & 0.12 & 35.78 & 1.44 & 0.02 & 47.98 & 1.69 & 2.26 & 0.01 \\
\hline Streptococcus & 0.00 & 0.38 & 0.00 & 0.00 & 2.55 & 0.28 & 0.00 & 0.61 \\
\hline \multicolumn{9}{|l|}{ Fungi } \\
\hline Aspergillus & 0.00 & 0.00 & 0.00 & 0.05 & 0.00 & 0.18 & 0.00 & 0.00 \\
\hline Candida & 0.00 & 0.51 & 0.00 & 0.00 & 24.42 & 0.00 & 0.00 & 1.90 \\
\hline Cladosporium & 0.00 & 0.00 & 0.01 & 0.00 & 0.00 & 0.00 & 0.00 & 0.00 \\
\hline Fusarium & 0.00 & 0.00 & 0.00 & 0.00 & 0.00 & 0.00 & 0.01 & 0.00 \\
\hline Geotrichum & 0.00 & 0.00 & 0.00 & 0.00 & 0.00 & 0.00 & 0.00 & 0.06 \\
\hline Trichosporon & 0.00 & 0.00 & 0.00 & 0.00 & 0.00 & 0.00 & 0.00 & 0.32 \\
\hline \multicolumn{9}{|l|}{ Unidentifiable } \\
\hline & 0.00 & 0.00 & 0.75 & 1.84 & 0.00 & 0.00 & 0.00 & 5.24 \\
\hline
\end{tabular}

Table 2. Growth results of drain samples. Drains $\mathrm{A}^{+}, \mathrm{B}^{+}, \mathrm{C}^{+}$, and $\mathrm{D}^{+}$were sink drain samples containing VIMpositive $P$. aeruginosa. Drains $\mathrm{E}^{-}, \mathrm{F}^{-}, \mathrm{G}^{-}$, and $\mathrm{H}^{-}$were sink drain samples that did not contain VIM-positive $P$. aeruginosa. Relative abundances are shown as percentages, and color-coded for low abundance (green; $<5 \%$ ), moderate abundance (yellow; 5-30\%), and high abundance (red; $>30 \%$ ); abundances of $0.00 \%$ (shown in white) indicate a complete absence of that genus in a sample. Genera are grouped in the categories Gram-positive or Gram-negative bacteria, fungi, and unidentifiable. A large amount of non-fermenting Gram-negative bacteria was discovered, so these are grouped separately. $\S=$ Achromobacter (Drain $\mathrm{A}^{+}$), Cupriavidus (Drain $\mathrm{G}^{-}$), and VIM-negative $P$. aeruginosa (Drain $\mathrm{H}^{-}$) grew in liquid culture media, and were uncountable. 
present in both (Table 2). Drain $\mathrm{F}^{-}$grew moderate relative abundances of Acinetobacter (19\%) and Sphingomonas (23\%), and a high relative abundance of Pseudomonas fluorescens group isolates ( $42 \%$ of colonies), specifically $P$. gessardii and P. proteolytica (Supplementary Table S3). Drain $\mathrm{G}^{-}$grew moderate relative abundances of Acidovorax (6\%), Chryseobacterium (11\%), Flavobacterium (15\%), Sphingobium (7\%), Sphingomonas (7\%), and P. aeruginosa group isolates (7\%), specifically P. oleovorans and P. pseudoalcaligenes (Supplementary Table S3); a high relative abundance of Stenotrophomonas ( $36 \%$ of colonies) was also recorded. Drain $\mathrm{H}^{-}$grew moderate relative abundances of Acinetobacter (14\%), Citrobacter (8\%), Sphingomonas (7\%), and P. fluorescens group isolates (16\%), specifically P. proteolytica, P. brenneri, P. rhodesiae, and P. koreensis (Supplementary Table S3); a high relative abundance of Delftia (37\% of colonies) was also recorded. Five percent of colonies from Drain $\mathrm{H}^{-}$were unidentifiable (Table 2). Except for Drains $\mathrm{B}^{+}$and $\mathrm{E}^{-}$, sink drain samples were populated by few Gram-positive bacteria and fungi (Fig. 2).

Microbial load and genus composition varied widely between samples, yielding between 471 and 18,904 distinct colonies, and between 8 and 20 identifiable genera. There was a small number of colonies that could not be subcultured from original cultures, suggesting that these microorganisms depended on polymicrobial coculture for survival or were very sensitive to certain culture conditions. In total, 44 genera were identified across all samples, and most could be identified to species level using MALDI-TOF MS (Supplementary Table S3). However, $16 \mathrm{~S}$ rRNA sequencing was necessary to identify the following to genus or species level: Cladosporium spp. (for Drain $\mathrm{C}^{+}$); Brevibacterium spp. and Microbacterium spp. (for Drain $\mathrm{D}^{+}$); Acetobacter indonesiensis, Flavobacterium tructae, Moraxella osloensis, and Sphingobium yanoikuyae (for Drain $\mathrm{F}^{-}$); Bacillus circulans, Cupriavidus spp., Pseudoxanthomonas mexicana, Sphingobium yanoikuyae, and Sphingomonas aerolata (for Drain $\mathrm{G}^{-}$); and Chryseobacterium spp., Chryseobacterium ureilyticum, and Clostridium algidixylanolyticum (for Drain $\mathrm{H}^{-}$).

Growth in broths and blood culture bottles was uncountable, and only used to document the presence/absence of microorganisms not found in solid media. The only genera that were exclusively cultivated in these conditions and not in solid culture media were Achromobacter and Cupriavidus (Table 2).

Furthermore, three culture conditions specialized in Pseudomonas selection were compared. Across all samples, cetrimide agar grew all non-aeruginosa Pseudomonas spp., except in Drain $\mathrm{G}^{-}$; however, P. aeruginosa was only detected in two out of four positive sink drain samples (Drains $\mathrm{A}^{+}$and $\mathrm{C}^{+}$) using this medium (Table 3). The CAZ-VAN enrichment broth (used to culture swabs for $b l a_{\mathrm{VIM}}$ PCR) grew all non-aeruginosa Pseudomonas spp., except in Drains $\mathrm{G}^{-}$and $\mathrm{H}^{-}$; VIM-positive P. aeruginosa was detected in three out of four positive sink drain samples (Drains $\mathrm{A}^{+}, \mathrm{B}^{+}$, and $\mathrm{C}^{+}$) using this medium (Table 3). Finally, Mueller Hinton (MH) agar made with tap water from the tested wards grew several water-associated microorganisms, including non-aeruginosa Pseudomonas spp. from all samples; VIM-positive P. aeruginosa from all four of the positive sink drain samples was also detected using this medium (Table 3).

$b^{b l a_{\mathrm{VIM}}}$ in other Gram-negative isolates. One hundred eighty-eight Gram-negative bacterial isolates, including $P$. aeruginosa, were screened for $b a_{\mathrm{VIM}}$ using PCR. Besides $P$. aeruginosa from positive sink drain samples, $P$. putida group isolates from Drain $\mathrm{A}^{+}$were also positive for $b l a_{\mathrm{VIM}}$. All other Gram-negative bacterial isolates were negative.

Legionella and Acanthamoeba in drain samples. Aliquots from each 1- and 5-min sonication fluid suspension were screened for Legionella spp. and Acanthamoeba spp. using PCR, but all samples were negative.

\section{Discussion}

In this study, a microbial culturomics approach was applied to eight hospital sink drain samples to determine the viable microbiota in drains with and without persistent VIM-positive P. aeruginosa. In these niches, cultures are often targeted toward MDR bacteria following an outbreak, and not toward all available microorganisms; the novelty of our study is that all cultivable microorganisms were examined. Moreover, to the best of our knowledge, culturomics has never been used to investigate the innate hospital environment before. Our data provide unique insights into the microbial compositions of sink drains and inlets. These sites are worrisome transmission sources because they are more accessible to the environment outside of the sink drain, and are coincidentally microbial hotspots from which MDR bacteria may disperse. Furthermore, P. aeruginos a colonization in siphons has mainly been reported at distal ends rather than within water distribution systems ${ }^{11}$, so targeting $P$. aeruginosa growth near drain inlets is critical for limiting environmental transmission of this bacterium.

Our experiments revealed the presence of a large number of Gram-negative non-fermenting bacteria in all eight drain samples. This group of bacteria includes P. aeruginosa, and is associated with waterborne transmission and nosocomial, opportunistic infections ${ }^{20,21}$. Overall, culturomics results suggest that the microbial compositions of our hospital's drains largely consisted of Acinetobacter, Pseudomonas, Sphingomonas, and Stenotrophomonas; these findings are consistent with previous studies identifying these bacteria as common inhabitants of healthcare water systems ${ }^{20-22}$. However, we showed that the abundances of these four genera were generally lower in VIM-positive sink drain samples; the exception at species level was $P$. aeruginosa, which was present in all VIM-positive samples, and in extremely low abundance in VIM-negative samples. Some Gram-negative non-fermenting bacteria were completely absent in VIM-positive samples: Acidovorax, Chryseobacterium, Flavobacterium, and Sphingobium. A possible explanation for this could be active or passive competition between these non-fermenters and P. aeruginosa in this niche ${ }^{23}$. Surprisingly, certain species we anticipated finding based on their contributions to nosocomial infections were not found in any sample, namely Klebsiella pneumoniae, Escherichia coli, and Staphylococcus aureus.

In addition, two VIM-negative sink drain samples (Drains $\mathrm{E}^{-}$and $\mathrm{H}^{-}$) and one VIM-positive sink drain sample with a low abundance of VIM-positive P. aeruginosa (Drain $\mathrm{B}^{+}$) contained Candida spp., especially $C$. 


\begin{tabular}{|c|c|c|c|c|}
\hline Drain sample & Genus & Cetrimide agar & CAZ-VAN enrichment broth & $\begin{array}{l}\text { Mueller Hinton agar with } \\
\text { hospital tap water }\end{array}$ \\
\hline \multirow{8}{*}{ Drain $\mathrm{A}^{+}$} & Acinetobacter & & & $x$ \\
\hline & Brevundimonas & & & $x$ \\
\hline & Citrobacter & & $x$ & $x$ \\
\hline & Delftia & & & $x$ \\
\hline & Enterobacter & & & $x$ \\
\hline & Pseudomonas aeruginosa & $x$ & $x$ & $x$ \\
\hline & Pseudomonas putida group & $x$ & $x$ & $x$ \\
\hline & Stenotrophomonas & & & $x$ \\
\hline \multirow{7}{*}{ Drain $\mathrm{B}^{+}$} & Bacillus & & & $x$ \\
\hline & Enterococcus & & & $x$ \\
\hline & Kocuria & & & $x$ \\
\hline & Micrococcus & & & $x$ \\
\hline & Pseudomonas aeruginosa & & $x$ & $x$ \\
\hline & Roseomonas & & & $x$ \\
\hline & Staphylococcus & & & $x$ \\
\hline \multirow{2}{*}{ Drain $\mathrm{C}^{+}$} & Enterococcus & & & $x$ \\
\hline & Pseudomonas aeruginosa & $x$ & $x$ & $x$ \\
\hline \multirow{6}{*}{ Drain $\mathrm{D}^{+}$} & Aspergillus & & & $x$ \\
\hline & Kocuria & & & $x$ \\
\hline & Microbacterium & & & $x$ \\
\hline & Micrococcus & & & $x$ \\
\hline & Pseudomonas aeruginosa & & & $x$ \\
\hline & Sphingomonas & & & $x$ \\
\hline \multirow{4}{*}{ Drain $\mathrm{E}^{-}$} & Candida & & $x$ & $x$ \\
\hline & Lactobacillus & & & $x$ \\
\hline & Micrococcus & & & $x$ \\
\hline & Staphylococcus & & & $x$ \\
\hline \multirow{6}{*}{ Drain $\mathrm{F}^{-}$} & Acidovorax & & & $x$ \\
\hline & Acinetobacter & & & $x$ \\
\hline & Micrococcus & & & $x$ \\
\hline & Pseudomonas fluorescens group & $x$ & $x$ & $x$ \\
\hline & Sphingomonas & & & $x$ \\
\hline & Stenotrophomonas & & & $x$ \\
\hline \multirow{7}{*}{ Drain $\mathrm{G}^{-}$} & Acidovorax & & & $x$ \\
\hline & Acinetobacter & & & $x$ \\
\hline & Chryseobacterium & & & $x$ \\
\hline & Flavobacterium & & $x$ & $x$ \\
\hline & Pseudomonas aeruginosa group & & & $x$ \\
\hline & Sphingobium & & & $x$ \\
\hline & Stenotrophomonas & & & $x$ \\
\hline \multirow{11}{*}{ Drain $\mathrm{H}^{-}$} & Acidovorax & & & $x$ \\
\hline & Acinetobacter & & & $x$ \\
\hline & Candida & & $x$ & \\
\hline & Citrobacter & & $x$ & $x$ \\
\hline & Delftia & & & $x$ \\
\hline & Micrococcus & & & $x$ \\
\hline & Pseudomonas fluorescens group & $x$ & & $x$ \\
\hline & Sphingomonas & & & $x$ \\
\hline & Stenotrophomonas & & & $x$ \\
\hline & Streptococcus & & & $x$ \\
\hline & Trichosporon & & & $x$ \\
\hline
\end{tabular}

Table 3. Growth in Pseudomonas selective media. Drains $\mathrm{A}^{+}, \mathrm{B}^{+}, \mathrm{C}^{+}$, and $\mathrm{D}^{+}$were sink drain samples containing VIM-positive P. aeruginosa. Drains $\mathrm{E}^{-}, \mathrm{F}^{-}, \mathrm{G}^{-}$, and $\mathrm{H}^{-}$were sink drain samples that did not contain VIM-positive P. aeruginosa. In the table, a cross mark means that growth was observed in the indicated condition for that genus. CAZ ceftazidime; VAN vancomycin. 
albicans (Table 2). P. aeruginosa and C. albicans have previously been shown to competitively secrete metabolites that inhibit virulence or growth in one another ${ }^{24}$; such behavior may be occurring in sink environments, but this has not yet been reported. C. albicans, among other fungi, are also capable of modifying the $\mathrm{pH}$ of their environments by secreting organic acids ${ }^{25}$; interestingly, acetic acid has been used before to successfully decolonize hospital sinks harboring VIM-positive $P$. aeruginos $a^{26}$. No study has yet investigated the ability of $P$. aeruginosa and Candida to coexist in hospital sinks, so it is of keen interest for us to determine if the presence or absence of Candida, as well as the aforementioned Gram-negative non-fermenting bacteria, inhibit colonization of VIMpositive $P$. aeruginosa in drains. It should be noted, however, that Candida are also capable of causing serious infections in hospitalized patients, and its presence in the innate hospital environment is worrisome as well. Nevertheless, microorganisms that produce acids may be useful probiotic candidates against environmental P. aeruginosa; besides Candida, the presences of Lactobacillus and Acetobacter in our negative drain samples (Drains $\mathrm{E}^{-}$and $\mathrm{F}^{-}$, respectively) are of particular interest (Table 2). An increasing number of reports propose using Bacillus spp., such as B. subtilis, B. megaterium, and B. pumilus, as probiotic cleansers in hospitals; they do not produce acids, but have been shown to secrete an enzyme that disrupts the extracellular polymeric substance (EPS) matrix in clinically-relevant biofilms $\mathrm{s}^{27,28}$. In our study, the same Bacillus spp. were found in some sinks, but in low loads; additionally, Bacillus were found in both positive and negative drain samples. While spores were specifically isolated using a thermic shock pre-culture condition, we did not microscopically confirm if Bacillus existed as spores in these samples. Therefore, it is possible that ungerminated Bacillus spores were missed by both the culture-dependent and culture-independent methods used. This is relevant, as Bacillus produce spores in response to unfavorable surroundings, and in one study, B. subtilis existed almost exclusively as spores in freshwater conditions ${ }^{29}$. The aforementioned probiotic sanitation method employed by Caselli et al. comprised a fixed concentration of Bacillus spores as well. Therefore, while Bacillus are less convincing as probiotic candidates for sinks based on our findings, we also cannot rule them out.

We also observed differences in culture media aimed at isolating Pseudomonas spp. (Table 3). Cetrimide agar is a culture medium selective for Pseudomonas spp., but $P$. aeruginosa was only detected on this medium in the two positive sink drain samples with the highest abundances of $P$. aeruginosa (Table 3). An in-house enrichment broth containing $2 \mathrm{mg} / \mathrm{l}$ ceftazidime and $50 \mathrm{mg} / \mathrm{l}$ vancomycin (CAZ-VAN) used by our laboratory for detecting VIM-positive $P$. aeruginosa was successful at detecting this microorganism in three of the positive sink drain samples, but not in the drain sample where P. aeruginosa was in lowest abundance (Drain $\mathrm{D}^{+}$) (Table 3). The most sensitive medium used to culture $P$. aeruginosa was Mueller Hinton $(\mathrm{MH})$ agar made with tap water from the tested wards, which cultured $P$. aeruginosa from all four positive sink drain samples (Table 3). This medium was created to determine if compounds added to the tap water in these wards-20-50 $\mu \mathrm{g} / \mathrm{l}$ silver and 400-600 $\mu \mathrm{g} /$ copper to inhibit Legionella growth-also affected the growth of $P$. aeruginosa. The success of this medium at detecting VIM-positive $P$. aeruginosa suggests that customizing culture conditions to mimic the environment of origin is an effective strategy for culturing this microorganism, even when in extremely low loads. It is also evident from Table 3 that this medium was successful at cultivating several genera from our drain samples, so in future culturomics studies targeting sinks, the number of culture conditions can be reduced when $\mathrm{MH}$ agar with tap water is used.

This study had some limitations. Firstly, we selected negative sinks based on our definition that VIM-positive $P$. aeruginosa had not been cultured from these sinks, even after exposure to patients carrying this bacterium; however, the exposure time and use of these sinks by culture-positive patients are unknown. Secondly, among the 179 culture conditions employed during culturomics, cell culture capable of isolating viruses and phages was not included. While viruses may have been present and influential on drain microbiota, and in light of the labor-intensiveness of culturomics, we decided to focus on cultivating bacteria and fungi only, as these are currently being used as probiotics. Thirdly, we based the purity of cultures on colony morphology, so some species may have gone unidentified if their morphologies were too similar in appearance to others. Fourthly, we did not measure biofilm-specific markers, such as those relevant to EPS formation, so it is unclear if biofilms were present on drain plugs at the moment they were removed; subtle differences in nutrient exposure and moisture between drain plugs, inlets, and siphons could have contributed to different biofilm compositions in these sites. Most importantly, uncultivable or difficult-to-culture microorganisms may have been missed, so culturomics cannot be considered a standalone method for microbiota analysis. This shortcoming can be overcome by using molecular techniques, such as 16S and 18S/ITS rRNA sequencing. One advantage of using culturomics over molecular techniques is that isolates are obtained in culture, and can be used for future analyses. Moreover, molecular methods are highly sensitive, and will amplify DNA of non-living microorganisms, providing no insight into the viability or interactions of microorganisms in a niche ${ }^{30}$. For these reasons, culturomics is a valuable addition to molecular methods for microbiota analysis, especially when the viability of microbiota is important.

In conclusion, the composition of VIM-positive P. aeruginosa reservoirs formed in hospital sink drains was until now an unexplored topic. We show that a high abundance of Gram-negative non-fermenting bacteria, including Acinetobacter, non-aeruginosa Pseudomonas spp., Acidovorax, Chryseobacterium, Flavobacterium, and Sphingobium, as well as Candida characterized sink drains where VIM-positive P. aeruginosa has never successfully formed environmental reservoirs. It is possible that these microorganisms inhibit persistence of $P$. aeruginosa by, for example, producing acids that prohibit long-term colonization. However, these results are a snapshot of the microbiota present at a single moment, and may produce different results if the same sink drains are re-cultured at a different moment, perhaps reflecting the microbiota of patients admitted to those rooms at the time. Furthermore, drain microbiota may be influenced by the frequency at which sinks are used, the composition of materials in the sinks and pipelines, exposure to nutrients and biocides, and other environmental factors, such as room temperature and humidity. Nevertheless, by examining positive and negative sink drains from different hospital rooms using a standardized culturomics approach, we were able to decipher patterns between environments where VIM-positive P. aeruginosa has been a successful colonizer, and environments 
where it has not, even though the number of included sink drain samples was low; the labor-intensiveness of experiments limited the number of sinks that we could analyze. Continuing research on sink drain composition should target a larger number of sinks, ideally from multiple hospitals, and combine culturomics with molecular methods. Additionally, the microbial consortia found should be tested in competition experiments, incorporating $\mathrm{pH}$ modulation, to draw more firm conclusions on which microorganisms enable or inhibit persistence of VIM-positive $P$. aeruginosa in sinks. These results may prove valuable in designing environmental probiotics that replace ineffective disinfectants to decolonize hospital sinks, or prevent formation of drain reservoirs. Due to increasing multidrug resistance and the prevalence of $P$. aeruginosa clone ST111 in hospitals, measures to prevent this microorganism from surviving in reservoirs, or to eradicate reservoirs that form, should be labeled high priority for infection prevention and control.

\section{Data availability}

Isolates and datasets generated by this study are available on request from the corresponding author.

Received: 12 February 2020; Accepted: 15 September 2020

Published online: 13 October 2020

\section{References}

1. Kizny Gordon, A. E. et al. The hospital water environment as a reservoir for carbapenem-resistant organisms causing hospitalacquired infections-a systematic review of the literature. Clin. Infect. Dis. 64, 1435-1444 (2017).

2. Carling, P. C. Wastewater drains: epidemiology and interventions in 23 carbapenem-resistant organism outbreaks. Infect. Control Hosp. Epidemiol. 39, 972-979 (2018).

3. Loveday, H. P. et al. Association between healthcare water systems and Pseudomonas aeruginosa infections: a rapid systematic review. J. Hosp. Infect. 86, 7-15 (2014).

4. Otter, J. A. et al. Surface-attached cells, biofilms and biocide susceptibility: implications for hospital cleaning and disinfection. J. Hosp. Infect. 89, 16-27 (2015).

5. Van der Bij, A. K. et al. First outbreak of VIM-2 metallo-beta-lactamase-producing Pseudomonas aeruginosa in the Netherlands: microbiology, epidemiology and clinical outcomes. Int. J. Antimicrob. Agents 37, 513-518 (2011).

6. Voor, A. F. et al. VIM-positive Pseudomonas aeruginosa in a large tertiary care hospital: matched case-control studies and a network analysis. Antimicrob. Resist. Infect. Control. 7, 32 (2018).

7. Van der Bij, A. K. et al. Metallo-beta-lactamase-producing Pseudomonas aeruginosa in the Netherlands: the nationwide emergence of a single sequence type. Clin. Microbiol. Infect. 18, E369-E372 (2012).

8. Oliver, A., Mulet, X., Lopez-Causape, C. \& Juan, C. The increasing threat of Pseudomonas aeruginosa high-risk clones. Drug Resist. Updat. 21-22, 41-59 (2015).

9. Hota, S. et al. Outbreak of multidrug-resistant Pseudomonas aeruginosa colonization and infection secondary to imperfect intensive care unit room design. Infect. Control Hosp. Epidemiol. 30, 25-33 (2009).

10. Kotay, S. M. et al. Droplet- rather than aerosol-mediated dispersion is the primary mechanism of bacterial transmission from contaminated hand-washing sink traps. Appl. Environ. Microbiol. 85, E01997-E2018 (2019).

11. Bédard, E., Prevost, M. \& Deziel, E. Pseudomonas aeruginosa in premise plumbing of large buildings. Microbiologyopen 5, 937-956 (2016)

12. Lagier, J. C. et al. Microbial culturomics: paradigm shift in the human gut microbiome study. Clin. Microbiol. Infect. 18, 1185-1193 (2012).

13. Kaeberlein, T., Lewis, K. \& Epstein, S. S. Isolating, "uncultivable" microorganisms in pure culture in a simulated natural environment. Science 296, 1127-1129 (2002).

14. van der Zee, A. et al. Spread of carbapenem resistance by transposition and conjugation among Pseudomonas aeruginosa. Front. Microbiol. 9, 2057 (2018).

15. Monsen, T., Lovgren, E., Widerstrom, M. \& Wallinder, L. In vitro effect of ultrasound on bacteria and suggested protocol for sonication and diagnosis of prosthetic infections. J. Clin. Microbiol. 47, 2496-2501 (2009).

16. Kobayashi, N., Bauer, T. W., Tuohy, M. J., Fujishiro, T. \& Procop, G. W. Brief ultrasonication improves detection of biofilm-formative bacteria around a metal implant. Clin. Orthop. Relat. Res. 457, 210-213 (2007).

17. Bjerkan, G., Witso, E. \& Bergh, K. Sonication is superior to scraping for retrieval of bacteria in biofilm on titanium and steel surfaces in vitro. Acta Orthop. 80, 245-250 (2009).

18. Templeton, K. E. et al. Development and clinical evaluation of an internally controlled, single-tube multiplex real-time PCR assay for detection of Legionella pneumophila and other Legionella species. J. Clin. Microbiol. 41, 4016-4021 (2003).

19. Qvarnstrom, Y., Visvesvara, G. S., Sriram, R. \& da Silva, A. J. Multiplex real-time PCR assay for simultaneous detection of Acanthamoeba spp., Balamuthia mandrillaris, and Naegleria fowleri. J. Clin. Microbiol. 44, 3589-3595 (2006).

20. Decker, B. K. \& Palmore, T. N. The role of water in healthcare-associated infections. Curr. Opin. Infect. Dis. 26, 345-351 (2013).

21. McGowan, J. E. Jr. Resistance in nonfermenting gram-negative bacteria: multidrug resistance to the maximum. Am. J. Med. 119, S29-36 (2006).

22. Williams, M. M., Armbruster, C. R. \& Arduino, M. J. Plumbing of hospital premises is a reservoir for opportunistically pathogenic microorganisms: a review. Biofouling 29, 147-162 (2013).

23. Bauer, M. A., Kainz, K., Carmona-Gutierrez, D. \& Madeo, F. Microbial wars: competition in ecological niches and within the microbiome. Microb. Cell 5, 215-219 (2018).

24. Méar, J. B. et al. Candida albicans and Pseudomonas aeruginosa interactions: more than an opportunistic criminal association?. Med. Mal. Infect. 43, 146-151 (2013).

25. Vylkova, S. Environmental pH modulation by pathogenic fungi as a strategy to conquer the host. PLoS Pathog. 13, E1006149 (2017).

26. Aspelund, A. S. et al. Acetic acid as a decontamination method for sink drains in a nosocomial outbreak of metallo-beta-lactamaseproducing Pseudomonas aeruginosa. J. Hosp. Infect. 94, 13-20 (2016).

27. Caselli, E. et al. Impact of a probiotic-based hospital sanitation on antimicrobial resistance and HAI-associated antimicrobial consumption and costs: a multicenter study. Infect. Drug Resist. 12, 501-510 (2019).

28. Kalpana, B. J., Aarthy, S. \& Pandian, S. K. Antibiofilm activity of a-amylase from Bacillus subtilis S8-18 against biofilm forming human bacterial pathogens. Appl. Biochem. Biotechnol. 167, 1778-1794 (2012).

29. Popova, L. Y. et al. Population dynamics of transgenic microorganisms in the different microecosystem conditions. Adv. Space Res. 27, 1571-1579 (2001).

30. Johani, K. et al. Characterization of microbial community composition, antimicrobial resistance and biofilm on intensive care surfaces. J. Infect. Public Health 11, 418-424 (2018). 


\section{Acknowledgements}

This research was funded by Erasmus University Rotterdam (EUR) Fellowship No. 105866. The authors would like to thank the Unit Infection Prevention for assisting in sample retrieval, and the Unit Diagnostics for their help with PCR, fungal identification, and the preparation of media.

\section{Author contributions}

J.P. designed and executed experiments, analyzed data, and wrote the manuscript. S.H. and S.R. executed experiments. C.K. analyzed data and provided feedback on the manuscript. M.V. and W.vW. helped obtain funding, and provided feedback on the data analysis and manuscript. J.S. obtained funding, designed experiments, analyzed data, and provided feedback on the data analysis and manuscript. All authors read and approved the final manuscript.

\section{Competing interests}

The authors declare no competing interests.

\section{Additional information \\ Supplementary information is available for this paper at https://doi.org/10.1038/s41598-020-73650-8.}

Correspondence and requests for materials should be addressed to J.A.S.

Reprints and permissions information is available at www.nature.com/reprints.

Publisher's note Springer Nature remains neutral with regard to jurisdictional claims in published maps and institutional affiliations.

(c) (1) Open Access This article is licensed under a Creative Commons Attribution 4.0 International License, which permits use, sharing, adaptation, distribution and reproduction in any medium or format, as long as you give appropriate credit to the original author(s) and the source, provide a link to the Creative Commons licence, and indicate if changes were made. The images or other third party material in this article are included in the article's Creative Commons licence, unless indicated otherwise in a credit line to the material. If material is not included in the article's Creative Commons licence and your intended use is not permitted by statutory regulation or exceeds the permitted use, you will need to obtain permission directly from the copyright holder. To view a copy of this licence, visit http://creativecommons.org/licenses/by/4.0/.

(C) The Author(s) 2020 